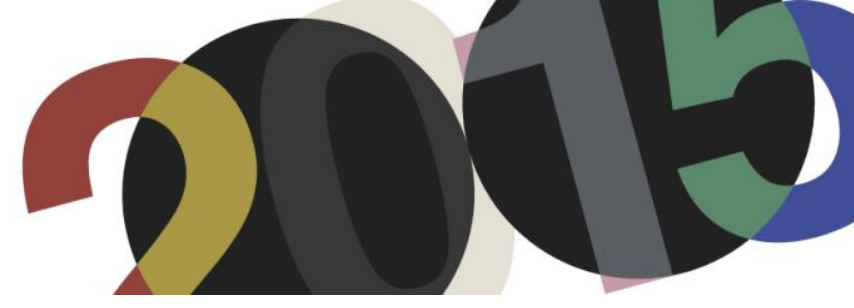

DOI: http://dx.doi.org/10.4995/LC2015.2015.659

\title{
Construcciones ambientales para el hábitat moderno: Le Corbusier y André Missenard (1937-57)
}

\author{
I. Requena, D. Siret \\ CRENAU - UMR CNRS-MCC-ECN 1563 Ambiances Architectures Urbanités. École Nationale Supérieure \\ d'Architecture de Nantes (Francia)
}

Resumen: Desde final de los años 30, la formulación de una nueva sensibilidad cultural, tecnológica y política hacia el clima, entendido en un sentido amplio, replanteó la relación entre el cuerpo humano y su entorno. El presente artículo confronta la obra de Le Corbusier ante dicha hipótesis de evolución del paradigma higienista previo. A partir de una investigación original en los archivos de la Fondation Le Corbusier, el objeto principal es el estudio de los intercambios teóricos y los aportes técnicos entre el arquitecto y André Missenard. El ingeniero, referente en el entorno científico, industrial y político de la época, resulta una pieza clave para entender la relevancia de la dimensión ambiental del hábitat propugnado por Le Corbusier durante los años 50. En particular, este trabajo aborda tanto los métodos de ideación (Grille Climatique) como su materialización espacial, técnica y sensorial (Unités d'habitation y Maison du Brésil). Las conclusiones del artículo muestran la evolución de los postulados habitacionales de Le Corbusier, que partiendo de la "Ville Radieuse", acabó por integrar las ideas de la "Science des climats artificiels" en el proyecto arquitectónico, buscando "Rétablir les conditions nature" en el hábitat moderno.

Abstract: Since the late-1930s, the emergence of a renewed cultural, technological and political approach towards the notion of climate reformulated the relationship between bodies and their milieux. This article analyses the works of Le Corbusier through the lens of this hypothesis of the hygienist paradigm's evolution. Based on an original research at the archives of the Fondation Le Corbusier, this paper focuses on the theoretical discussions and the technical collaboration of the architect with André Missenard. This engineer, a key figure in the scientific, industrial and political environment of his time, played a main role to understand the relevance of the ambient dimension in Le Corbusier's housing proposals in the 1950s. In particular, this paper deals with the design methods (Grille Climatique), as well as their spatial, technical and sensory materialization (Unités d'habitation and Maison du Brésil). The conclusions of the article show the evolution of Le Corbusier's postulates, which beginning from his early proposal for the "Ville Radieuse", managed to integrate the contributions of the "Science des climats artificiels" in architectural design looking for "Rétablir les conditions nature" in modern housing.

Palabras clave: André Missenard; climas artificiales; control ambiental; Le Corbusier. Keywords: André Missenard; artificial climates; thermal ambiances; Le Corbusier.

\section{Introducción: control ambiental y modernidad}

Desde finales del s. XIX, el enfoque higienista en medicina, arquitectura e ingeniería marcó la manera de construir los espacios destinados al trabajo, la salud o a la habitación. Diversas investigaciones actuales ${ }^{1}$ muestran la importancia en este periodo de la accesibilidad de luz solar y aire limpio como métodos de purificación ambiental en un sentido medicinal, social e incluso moral. No obstante, la constatación del aumento

\footnotetext{
${ }^{1}$ En particular: Campbell, M., What Tuberculosis Did for Modernism; Hobday, R., The Light Revolution; Siret, D., Les sensations du soleil dans les théories architecturales et urbaines.
} 
de la polución en el medio urbano ${ }^{2}$, entre otros aspectos, hizo aflorar a principios del s. XX nuevos ingenios técnicos que prometían purificar el aire del interior de los edificios. Sirvan de ejemplo el Climatogène de Bontemps (1901), la Air-conditioning unit de Carrier (1902) o el "Appareil pour laver l'air" de Richet (1910). Estos dispositivos nos indican el comienzo de una etapa donde las nuevas técnicas de control ambiental, unidas a los estudios fisiológicos sobre el sistema termorregulativo del cuerpo humano y a un medio social e industrial en busca de atmósferas interiores asépticas y reguladas, produjeron nuevas derivas del pensamiento higienista que tuvieron su traducción en la arquitectura de décadas posteriores.

Durante la primera mitad del s. XX, tal como Fleming y Jankovic indican ${ }^{3}$, dicho efecto se manifestó a través de una mayor relevancia científica y cultural de la noción de clima. Por una parte, al clima a gran escala, capaz de influir sobre la sociedad, la técnica y la cultura, como revelan por ejemplo la publicación de Civilization and Climate de Huntington (1924), la creación de la American Air Force Weather Service (1937) o la fundación de la World Meteorological Organization WMO de las Naciones Unidas (1951).

Por otra parte, el clima a escala del cuerpo humano, así como su control mediante las técnicas modernas. Apoyándose en la analogía del cuerpo-maquina, productor e intercambiador de energía con su medio, numerosos médicos, economistas, físicos, geógrafos e ingenieros ${ }^{4}$ desarrollaron investigaciones interdisciplinares que alimentaron la ambición de construir climas artificiales a medida. Una primera aproximación se construyó según el concepto de "air-conditioning (aire acondicionado)", definido por Carrier como el "control de las condiciones

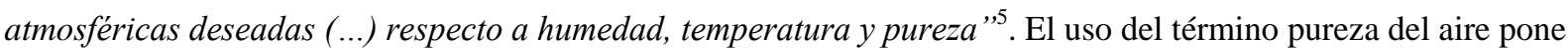
de manifiesto la continuidad entre sus objetivos iniciales y los del pensamiento higienista. Superados los debates heredados sobre la concentración de $\mathrm{CO}_{2}$ y sus efectos en la salud o en la eficiencia de trabajadores y escolares, la investigación científica se orientó hacia el análisis de la percepción sensorial individual in vitro de ambientes térmicos. La nueva meta era encontrar las condiciones ambientales de mayor eficiencia biológica y mental, considerando así al cuerpo humano como un simple receptor de estímulos pasivo, cuya situación ideal se encontraba en la neutralidad sensorial o la ausencia de estimulación ambiental. El avance tecnológico y comercial del aire acondicionado reconfiguró la percepción social de espacios como salas de cine, galerías comerciales o edificios de oficinas, cuyo atractivo residía además en sus cualidades térmicas.

A final de los años 20, Le Corbusier se hizo eco del mecanismo de ventilación Aération ponctuelle del ingeniero Gustave Lyon (1857-1936) con el objetivo de desarrollar su Respiration exacte. Combinada con un sistema de aislamiento activo, el Mur neutralisant, inspirado a su vez en la Maison isotherme de Raoul Decourt (1925), la propuesta climático-arquitectónica pretendía encarnar la utopía moderna de fabricar "todo el año aire exacto, renovado, desinfectado, exactamente humidificado y llevado a la temperatura constante de $18{ }^{\circ} \mathrm{C}$ " . Proyectos como el Centrosoyuz (Moscu, 1928) o la Cité de Refuge (Paris, 1929) fueron reflejo de un punto de vista que ya estaba presente de manera conceptual en Vers une architecture (1923): "una casa que sea ese límite humano, que nos envuelve, que nos separe del fenómeno natural antagonista, que nos de nuestro medio humano, a

\footnotetext{
${ }^{2}$ Simonnet, C., Brève histoire de l'air.

${ }^{3}$ Fleming, Roger; Jankovic, Vladimir: Rivisiting Klima.

${ }^{4}$ Ackermann, M., Cool Comfort: America's Romance with Air-Conditioning.

${ }^{5}$ Cooper, G., Air-Conditioning America: Engineers and the Controlled Environment, 1900-1960.

${ }^{6}$ Cita original: "fabriquer toute l'année de l'air exact, dépoussiéré, désinfecté, exactement humidifié et porté à température constante de $18^{\circ}$ environ". Cf. Le Corbusier, La ville radieuse : eléments d'une doctrine d'urbanisme pour l'équipement de la civilisation machiniste, p. 42.
} 
nosotros, los hombres ${ }^{\prime, 7}$. Tal como Torres $\mathrm{Cueco}^{8}$ señala, para Le Corbusier esta casa completamente regulada constituía un agente protector frente a un ambiente natural desordenado que dificultaba el desarrollo de la vida humana.

En paralelo al air-conditionning, profesionales de diverso ámbito defendieron una segunda tesis basada en las mismas bases científicas: la evolución de las nuevas técnicas de control ambiental permitiría crear en espacios interiores auténticos Climats artificiels en toda su complejidad, cuya interacción con el cuerpo humano sería aprovechada positivamente. La atmosfera interior no pretendía remplazar al clima natural, sino construirse íntimamente ligada a él y modularlo en sus condiciones más extremas. Desde los años 20, diversas aportaciones reflejaron esta articulación, por ejemplo la patente de vidrio Vitaglass permeable a los rayos UVA ${ }^{9}$, los ventiladores de velocidad variable y la ionización del aire ${ }^{10}$ o incluso la vacunación aérea. En definitiva, los Climats artificiels proponían una noción de atmósfera interior aumentada que, conservando las características de heterogeneidad y variabilidad del clima natural, permitiría mejorar la eficiencia biológica del Homme Nouveau.

En 1937 el ingeniero francés André Missenard (1901-1989), colaborador de Le Corbusier desde 1942, acuñó por primera vez el término de la Science des climats artificiels. Missenard tenía como objetivo devolver al ser humano a unas condiciones habitables próximas al clima natural que, según él, resultaba fisiológicamente idóneo para el desempeño de sus tareas vitales y sociales. Por lo tanto: "para la gran mayoría de seres humanos aclimatados a su hábitat, la solución mas racional, aunque parezca paradójico, sería cambiar su clima natural

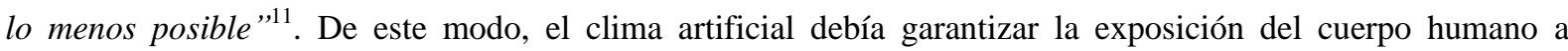
variaciones ambientales semejantes a las del clima exterior, lo cual impediría el acomodamiento del sistema termorregulador y reforzaría la respuesta inmunológica, haciéndolo más vigoroso. Missenard defendió que las técnicas de control ambiental debían ir más allá de la mera regulación térmica, debiendo aspirar a la creación de climas interiores $^{12}$. La Science des Climats artificiels se alineaba técnicamente con el término de climatización empleado en el mundo anglosajón: "conjunto de procedimientos que permiten realizar, en un local, un clima artificial caracterizado por el nivel de la sensación térmica, la pureza del aire, el nivel de la sensación sonora, la presencia de iones, la presión, etc ${ }^{\prime \prime 13}$. En definitiva, el clima artificial articuló los conocimientos de diversas disciplinas interesadas en las atmósferas del hábitat cotidiano y su papel en la construcción del proyecto de la modernidad.

\footnotetext{
${ }^{7}$ Cita original: "une maison qui soit cette limite humaine, nous entourant, nous séparant du phénomène naturel antagoniste, nous donnant notre milieu humain, à nous hommes". Cf. Le Corbusier, Vers une architecture.

${ }^{8}$ Torres Cueco, J., Pensar la arquitectura: Mise au point de Le Corbusier, p. 103.

${ }^{9}$ Sadar, J., The Healthful Ambience of Vitaglass: Light, Glass and the Curative Environment.

${ }^{10}$ Cooper, G., Ob. Cit.

${ }^{11}$ Missenard, A., L'homme et le climat.

${ }^{12}$ Missenard, A., Le conditionnement des locaux.

${ }^{13}$ Ibidem.
} 

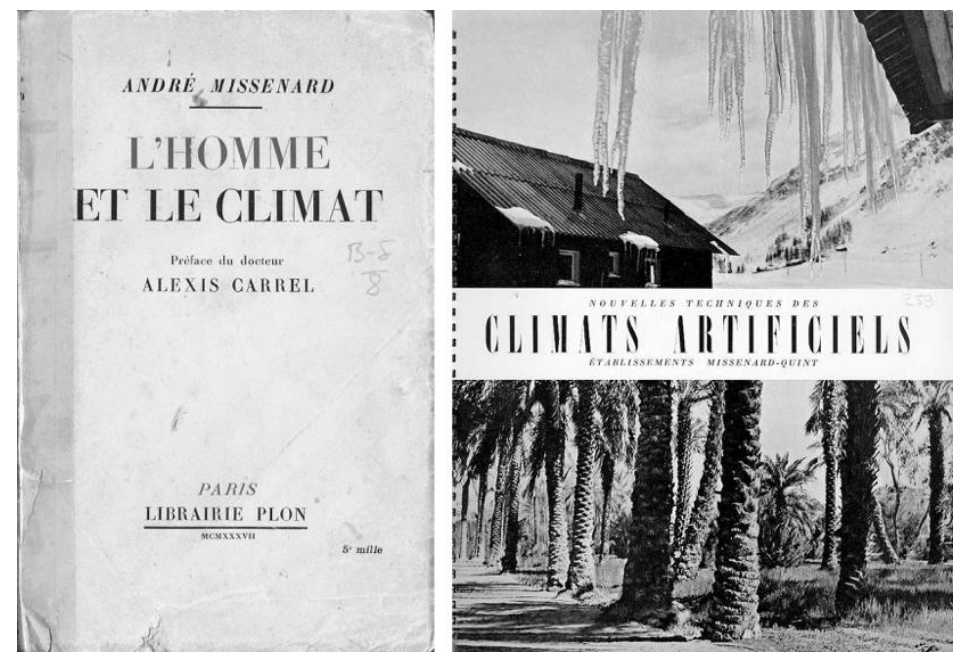

1. L'homme et le climat de André Missenard, prologado por Alexis Carrel, y tríptico publicitario de Ets Missenard-Quint en 1957 (FLC Q1-15-259). Fuente: ( F FLC-ADAGP.

En este contexto, la noción de clima transcendió su dimensión climatológica vinculándose a conceptos de naturaleza cultural o política, como el confort, la eficiencia o la ergonomía. Si bien el primer aspecto es visible en los trabajos de Corbin o de la Soudière ${ }^{14}$, el segundo ha sido especialmente subrayada por Sloterdijk ${ }^{15}$. La capacidad de controlar el clima a pequeña escala redefinió tanto los modos de ocio, de trabajo y de habitación propugnados por la modernidad, como los ambientes donde estas actividades debían llevarse a cabo. La arquitectura de la vivienda, en consecuencia, sirvió para operar una utopía climático-terapéutica de amplias implicaciones sociales. Reflejo de ello fue la introducción de la temática Climats artificiels en los comités pluridisciplinares de vivienda de la Sociedad de Naciones y de la Fondation Française pour l'Etude des Problèmes Humains ${ }^{16}$. El interés de Le Corbusier por estas cuestiones quedó patente en sus continuos intercambios y colaboraciones con médicos e ingenieros especializados (thermiciens) desde final de los años 30 hasta final de los 50, los cuales se detallan a continuación.

\section{Le Corbusier y los climas artificiales}

En su publicación L'Homme et le climat (1937), Missenard indicó por primera vez la necesidad de asentar las bases de una nueva disciplina: la Science des climats artificiels. A modo de manual el libro realiza un estado del arte de los fenómenos ambientales conocidos y sus efectos sobre la salud, la productividad o el comportamiento humano. Aunque Le Corbusier no conocería personalmente a Missenard hasta 1942, su biblioteca personal conservada en la FLC contiene una edición de L'Homme et le climat de 1937. El libro, posiblemente su primer contacto directo con las ideas de Missenard, revela a través de copiosas anotaciones el interés del arquitecto por la dimensión ambiental en la arquitectura y el urbanismo modernos. Además, el documento constata su intención de vincular los postulados técnico-científicos de Missenard con el proyecto de la Ville Radieuse (1934), instrumentalizando afirmaciones sobre el soleamiento, la ionización del aire, la ventilación natural o el apoyo a un modo de vida físicamente activo, a favor de un nuevo urbanismo.

\footnotetext{
${ }^{14}$ Véanse: De la Soudière, M., Au bonheur des saisons; Corbin, A., La pluie, le soleil et le vent.

${ }^{15}$ Sloterdijk, P., Esferas III - Espumas, Esferología plural.

${ }^{16}$ Marino, G. « Some Like It Hot ! » Le confort physiologique et ses dispositifs dans l'architecture du XXè siècle.
} 
Otra punto de acceso a las ideas subyacentes en la noción de clima artificial fue el círculo social común con Missenard, con personalidades como el biólogo y cirujano Alexis Carrel (1873-1944), premio nobel en medicina (1912), convencido defensor del eugenismo ${ }^{17}$ y presidente de la Fondation Française pour l'Etude des Problèmes Humains. También cabe destacar la figura de Pierre Winter (1891-1952), médico, biólogo e higienista francés, igualmente unido al movimiento eugenista, que fue amigo y vecino de Le Corbusier en el 24 rue Nungesser et Coli. El doctor era un convencido de la importancia de la arquitectura para la salud de la sociedad moderna y militaba por el enfoque ambiental de Missenard sobre el medio construido.

En Junio de 1937, Winter realizó una intervención en el V CIAM en Paris, titulada "Logis et loisirs (vivienda y ocio)". La ponencia recogió de manera general los requerimientos medicinales exigidos a las soluciones habitacionales modernas, dado que, en su opinión, para los médicos era imposible "cumplir correctamente su misión de 'reducir las enfermedades al mínimo estricto, construir cuerpos robustos y harmoniosos sin viviendas saludables, sin ocio organizado, sin la reconstrucción de las fabricas, de las oficinas, sin la creación de todo el equipamiento de salud" "18. Winter basaba su propuesta en la idea de obtener el máximo aprovechamiento de los beneficios biológicos del clima del lugar sobre el cuerpo humano, corregido mediante la arquitectura en caso de ser necesario. Por ejemplo, refiriéndose al soleamiento, Winter destacó la obligación de maximizar la captación de luz natural evitando la orientación Norte o aplicando correcciones arquitectónicas en caso necesario. Según su opinión la luz en la ciudad moderna estaba enferma: "disminución de rayos U.V. (ineficacia de la helioterapia urbana), disminución de los infrarrojos (calorificos) y perdida de elementos vitalizantes (...) El pan de verre, en la ciudad actual contaminada, es lo mejor que podemos desear; para las ciudades con aire puro, daremos todas las facilidades para el contacto directo entre la piel y la luz. "19 Sin embargo, aparte de indicaciones generales sobre orientación solar o ventilación, su proyecto no entraba en el detalle ni científico ni técnico sobre su materialización.

Como veremos a continuación, la relación profesional entre Le Corbusier y Missenard aportó un mayor grado de concreción en la dimensión técnica y fisiológica de los climas artificiales, asociándolo al uso de estrategias o dispositivos arquitectónicos. En consecuencia, su trabajo colaborativo durante los años 50 se convirtió en un laboratorio de soluciones ambientales para el hábitat moderno ${ }^{20}$.

No obstante, si bien Le Corbusier y Missenard parecían coincidir en los principios esenciales, sus puntos de vista divergían en cuanto a la manera de materializarlos. El trabajo de Le Corbusier intentaba acercar a los habitantes a unas condiciones naturales idealizadas, lo cual podía ocurrir dentro o fuera de la arquitectura, o como él mismo lo dijo en su articulo Aux approches d'une synthèse ${ }^{21}$, hacer que a través de la arquitectura moderna "les conditions de nature sont reconquises !’. Al contrario, Missenard pensaba que el clima artificial no debía ni suplir ni imitar el contacto real con la naturaleza, enfoque que le llevó a criticar la arquitectura de Le Corbusier: “es preocupante que los ventanales alargados de Le Corbusier, 'incorporando el paisaje en la vivienda', creen la peligrosa ilusión de la vida al aire libre ${ }^{, 22}$.

\footnotetext{
${ }^{17}$ Su libro L'Homme, cet inconnu publicado en 1935 se convirtió en un manifiesto reconocido internacionalmente.

${ }^{18}$ Winter, P., "Logis et loisirs", Junio 1937, FLC D2.11.557.

${ }^{19}$ Winter, P., "Logis et loisirs", Junio 1937, FLC D2.11.561.

${ }^{20}$ Requena-Ruiz, I.; Siret, D. Experiments on Thermal Comfort and Modern Architecture.

${ }^{21}$ Le Corbusier, Aux approches d'une synthèse. Aboutissement de vingt années consacrées à la recherche d'une doctrine du domaine bâti.

${ }^{22}$ Missenard, A., A la recherche de l'homme, p. 2.
} 


\section{La Grille Climatique: la regulación climática y el hábitat moderno}

La colaboración profesional Le Corbusier-Missenard se inició de forma efectiva tras su vuelta del segundo viaje a Chandigarh (India) en Noviembre de 1951. Le Corbusier escribió al ingeniero en busca de consejo experto, dado que la nueva serie de encargos en la India suponían un desafío de adaptación a un clima distinto. Consciente de la importancia del clima en estos proyectos, el arquitecto aprovechó el viaje para solicitar la información climatológica al Institute of Fundamental Research of Bombay ${ }^{23}$. Su carta a Missenard revela una ambición mayor: desarrollar un método de ideación que le permitiera obtener condiciones ambientales idóneas en la arquitectura para cualquier clima. Dado que la rigidez de las normativas en los países europeos dificultaban la experimentación a este respecto, Le Corbusier concebía sus proyectos en la India como una oportunidad de explorar otras soluciones de control ambiental: "Las reglamentaciones en relación a esta nueva capital implican la toma de decisiones respecto a las leyes de higiene (de diferente naturaleza a nuestras reglamentaciones conformistas y fruto de todos los compromisos) ${ }^{\text {"24 }}$.

El proceso de concretización del método se continuó de manera intensiva con la ayuda del ingeniero griego Iannis Xenakis (1922-2001), quien estableció el "Programa de estudios de las condiciones climáticas óptimas y de los medios arquitectónicos de corrección” climático" (temperatura, humedad relativa, velocidad del aire y temperatura de los muros) que determinarían "las variaciones ambientales óptima y teórica para conservar o incrementar el mejor rendimiento VITAL",26. El 20 de diciembre de 1951, Xenakis dibujó un diagrama titulado "Grille d'hygiène climatique”,27, aunque el término higiene se eliminó en diagramas posteriores ${ }^{28}$. La evolución de la terminología utilizada evidencia la influencia de Missenard en su elaboración" ${ }^{29}$. En paralelo, Samper elaboró un plano llamado "Esquisse générale

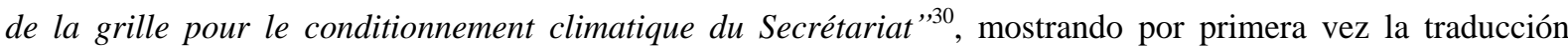
arquitectónica de los criterios físicos y fisiológicos indicados en la Grille Climatique.

A fecha 31 de enero de $1952^{31}$, el equipo de proyecto documentó el método de ideación en el dosier Grille Climatique, el cual además se complementó con un catálogo de soluciones aplicado en el proyecto de viviendas Maisons des péons $110 \mathrm{~m}^{2}$ (1952) en Chandigarh. Los autores lo describieron como un modo de visualización que permitiría enumerar, coordinar y analizar los datos climatológicos de un emplazamiento definido, con el objetivo de guiar la investigación arquitectónica hacia soluciones mas próximas a las necesidades de la biología

\footnotetext{
${ }^{23}$ Le Corbusier, carta a Pran Nath Thapar, 9 de Diciembre de 1951, FLC P2.17.183.

${ }^{24}$ Le Corbusier, carta a André Missenard, 3 de Diciembre de 1951, FLC E2.16.14. Cita original: "Des réglamentations concernant cette nouvelle capitale impliquent des decisión à prendre concernant les lois de l'hygiène (de tout autre natura que nos règlamentations conformistes et fruits de tous les compromis)".

${ }^{25}$ Xenakis, I., "Programme d'études des Conditions Climatiques Optima et des Moyens Architecturaux de correction," 16 de Diciembre de 1951, FLC P2.1.1.

${ }^{26}$ Ibidem. Cita original: "les variations optima et théoriques de l'ambiance pour conserver ou accroître le meilleur rendement VITAL".

${ }^{27}$ Denominación original: "Grille d'Hygiène Climatique", cf. Le Corbusier, carta a Jane Drew, 20 de Diciembre de 1951, FLC, G2.11.26.

${ }^{28}$ Le Corbusier, carta a Jane Drew, 21 de Diciembre de 1951, FLC P2.1.9.

${ }^{29}$ Siret, D. Grille Climatique, 1951.

${ }^{30}$ Samper, G., "Esquisse générale de la grille pour le conditionnement climatique du Secrétariat", 21 de Diciembre de 1951, FLC 2642.

${ }^{31}$ Atelier Le Corbusier, "Grille climatique”, 31 de Enero de 1952, FLC P2.1.3.
} 
humana. A través de estrategias y dispositivos arquitectónicos diversos, su interés residía en la regulación y corrección efectiva de los excesos del clima para crear las condiciones de bienestar y el confort ${ }^{32}$.

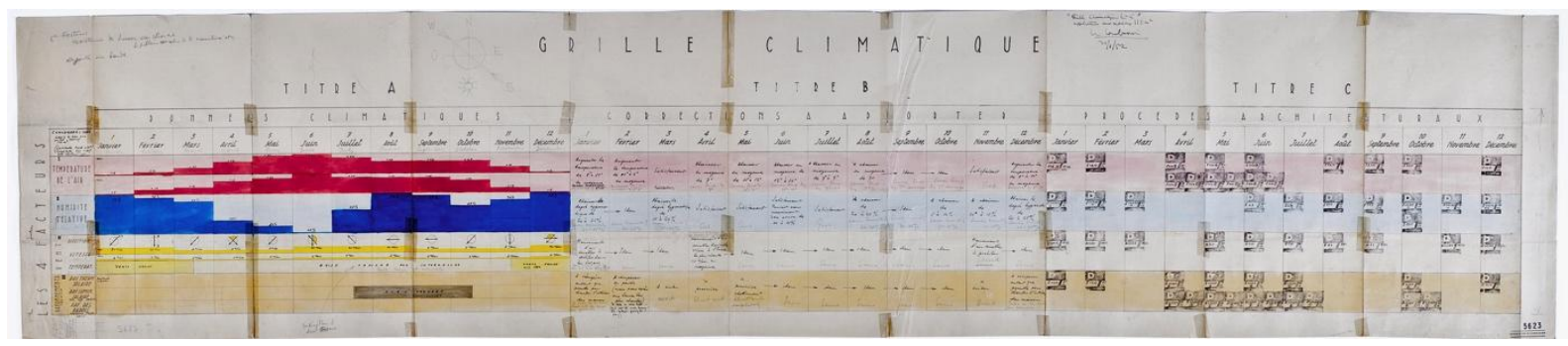

2. Grille Climatique según los datos climatológicos de Chandigarh (FLC 5623). Fuente: @ FLC-ADAGP.

El esquema definitivo quedó compuesto por tres columnas. La primera, titulada "Datos climáticos" (con anterioridad "Condiciones de ambiente”), recogía la información climatológica correspondiente a los cuatro factores ambientales propuestos originalmente por Missenard: temperatura del aire, humedad relativa, dirección y velocidad del viento, así como soleamiento y radiación térmica de las construcciones. La segunda columna fue llamada "Correcciones a ofrecer" (previamente "Correcciones en vista del confort y el bienestar"), remplazando la expresión del "mejor rendimiento vital” de la versión precedente. Según las instrucciones escritas, esta fila debía ser rellenada por un "físico-biólogo" "33, quien determinaría un programa de condiciones ambientales que serviría de apoyo a la intervención de los arquitectos. La tercera columna fue titulada "Procedimientos arquitectónicos" (antes "Soluciones arquitectónicas"), teniendo como cometido indicar la existencia de una solución técnico-formal aconsejada.

La identificación de dichas propuestas se hizo mediante un sello con la letra D seguida de un código numérico, haciendo alusión a un conjunto de dieciséis croquis específicos personalmente realizados y firmados por Le Corbusier $^{34}$. Por medio de una sección y una planta para cada propuesta, los arquitectos ilustraron las soluciones primarias y sus combinaciones: control solar, regulación de la humedad, control de la ventilación cruzada y construcción con alta inercia térmica. El proyecto elegido, las Maisons des péons $110 \mathrm{~m}^{2}$, se trataba de un conjunto de viviendas unifamiliares en hilera basado en otro proyecto previo no construido de prototipos de viviendas para el plan urbano de Bogotá (Colombia, 1950). El principal valor de este trabajo no realizado en la India fue, precisamente, servir de ejemplo para la aplicación de estrategias pasivas de climatización en el hábitat social moderno.

\footnotetext{
${ }^{32}$ Ibidem.

${ }^{33}$ En el primer y único ejemplo de la Grille Climatique, fue Missenard quien rellenó estas notas en tanto que "physicobiologiste". Cf. Missenard, "Corrections désirables dans les locaux," 16 de Enero de 1952, FLC, P2.1.27.

${ }^{34}$ Conservados en el conjunto de dibujos con la cota FLC 5627.
} 

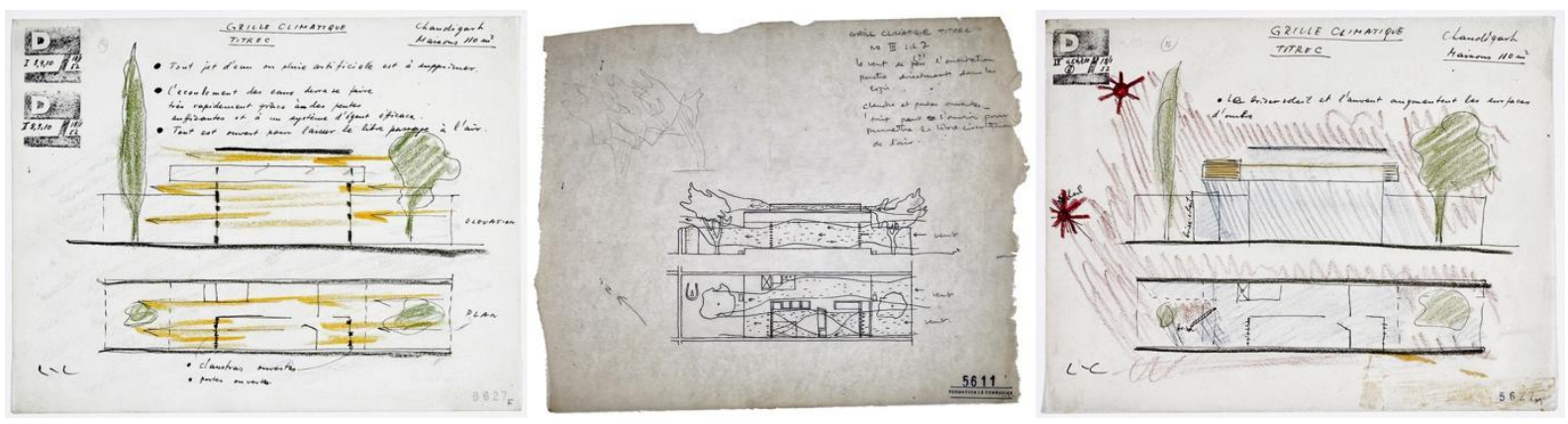

3. Serie de croquis adjuntos a la Grille Climatique. Fuente: () FLC-ADAGP.

Finalizado el periodo intensivo de reflexión metodológica, la Grille climatique desapareció de los trabajos del Atelier 35S, aunque Le Corbusier insistió con posterioridad en su interés para Chandigarh: "Pero esta Grille Climatique no ha sido tenida en consideración en Chandigarh y yo lo lamento amargamente (...) Habiendo sido perdida esta Grille yo estoy dispuesto a hacer establecer una nueva y a enviarla a Chandigarh si me lo solicitan ${ }^{, 35}$. No obstante, las estrategias de intervención propuestas en las citadas viviendas formaron parte del argumentario proyectual del Atelier $35 S$ en sus grandes proyectos públicos (e.g., Secretariado Chandigarh 1950, Alta Corte Judicial Chandigarh 1950, Palacio de los Hilanderos Ahmedabad 1956), pero también en sus proyectos de vivienda individual (e.g., Villa Sarabhai Ahmedabad 1955, la Villa Shodan Ahmedabad 1956) y colectiva (e.g., Convento de la Tourette Eveux-sur-l'Arbresle 1959 o la Casa de Brasil Paris 1959).

\section{Sistemas radiantes y prototipos climático-espaciales}

A lo largo de los sucesivos estados del arte realizados por Missenard para alimentar el corpus teórico de la Science des Climats Artificiels, los estudios de las técnicas de regulación ambiental y la reacción fisiológica desencadenada mostraron el interés del ingeniero por los sistemas radiantes. Una elección que venía justificada por diversos motivos: la baja temperatura de calefacción que minimizaría los movimientos del aire, reduciendo la difusión de microbios en comparación a otros sistemas; la libertad de instalación respecto a las particiones y los cerramientos; la posibilidad de mantener temperaturas de aire bajas, supuestamente más saludables para los pulmones; y la durabilidad de la instalación, dado que quedaría protegida dentro del hormigón ${ }^{36}$. Paradójicamente, Missenard era escéptico a la tesis defendida por algunos de sus colegas ingenieros, que equiparaba la calefacción radiante al modo natural por el cual recibimos la radiación solar desde el cielo (calefacción en el techo) o desde el terreno (suelo radiante). Aunque aceptaba que la radiación se asemejaba en mayor medida a la manera natural en la que el cuerpo humano recibe la energía calorífica, no coincidía en que su artificialización tuviera las mismas propiedades que la radiación luminosa y calorífica del Sol ${ }^{37}$.

Durante los años 40, Missenard desarrolló una patente de calefacción por suelo radiante: el Système Missenard. El método consistía en una instalación de tuberías de diámetro medio integradas en la losa de hormigón, convirtiendo el elemento estructural a la vez en calefactor. Sin embargo, hacia final de la década la compañía Ets Missenard-Quint no solo explotaba su patente, sino que también era concesionaria para Francia de la patente de

\footnotetext{
${ }^{35}$ Le Corbusier, carta a Varma, 22 de Mayo de 1956, FLC P1.10.303. Cita original: "Mais cette Grille Climatique n'a pas été prise en consideration à Chandigarh et je le regrette amérement (...) Cette Grille ayant été perdue je suis prêt à en faire établir une nouvelle et à l'envoyer à Chandigarh si on me la demande".

${ }^{36}$ Missenard, A., A la recherche du temps et du rythme.

${ }^{37}$ Missenard, A., Le Chauffage et la rafraichissement par rayonnement.
} 
superficies radiantes de la multinacional inglesa Richard Crittall \& Co, que a su vez era concesionaria del Système Missenard para el resto de Europa ${ }^{38}$. A diferencia de la licencia Missenard, la patente de Crittall se realizaba mediante tuberías de pequeño diámetro y se instalaba en la capa superficial de techos o muros. La conexión entre las dos empresas manifiesta su disposición a emprender en técnicas modernas de acondicionamiento ambiental, así como su interés en la relación entre la técnica y la arquitectura modernas. Sirva de ejemplo el hecho de que los sistemas de Richard Crittall \& Co fueron utilizados, entre otros, por el ingeniero finés Carl Rosenqvist, colaborador de Alvar Aalto ${ }^{39}$. Finalmente, cabe destacar que, tras varios debates entre los ingenieros y el Atelier $35 S$ relativos a la calefacción de las Maisons Jaoul (Neuilly-sur-Seine, 1951-5), el menor coste del Système Missenard provocó que fuera el único ejecutado en las obras de Le Corbusier, como la Maison du Brésil (Paris, 1953-59) o las Unités d'habitation desde Rezé (1949-56).
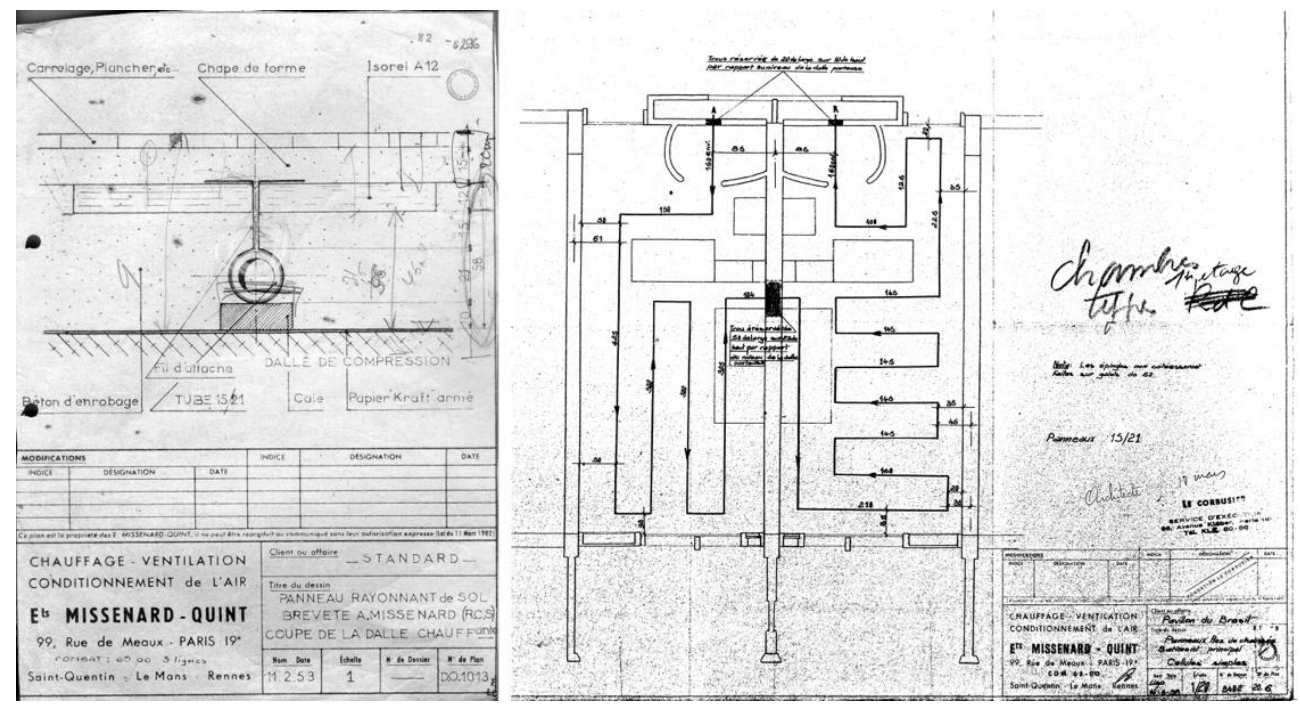

4. Esquema del Système Missenard y plano instalación de planta baja en la Casa de Brasil. C FLC-ADAGP.

La adaptación de un sistema de calefacción radiante, basado en los principios de gran inercia térmica y temperatura constante en su periodo activo, a los requisitos de variabilidad exigidos según los criterios de la Science des climats artificiels, fue abordada por Missenard de diversas maneras. En primer lugar, la diferenciación de los circuitos de alimentación en fluido calorífico según la orientación y las pérdidas térmicas de la zona, estrategia empleada en las Unités d'habitation a partir de Rezé o en la Maison du Brésil. Si tomamos de ejemplo el caso de Rezé, la instalación cuenta con cinco circuitos de calefacción diferentes no delimitados a cada vivienda sino al grado de exposición. Los circuitos se definieron entonces como: fachada este, fachada oeste, fachada sur, planta primera y planta ático. En segundo lugar, la temperatura del fluido calorífico se regulaba de manera continua a través de una sonda interior y otra exterior para cada circuito, especialmente desarrolladas por Ets Missenard-Quint ${ }^{40}$ para permitir la adaptación a las oscilaciones térmicas exteriores. La regulación se realizaba de manera centralizada según los criterios establecidos por la ingeniería, no permitiendo la interacción con los habitantes. En tercer lugar, la temperatura de cada espacio se pensó en base al uso del

\footnotetext{
${ }^{38}$ Prueba de la importancia de este acuerdo es su presentación en el encabezado del papel oficial de Ets Missenard-Quint en los años 50 con la leyenda "Concessionnaire des Brevets Internationaux de Chauffage par Rayonnement. Licence Crittall". Cf. Bernard Morel, carta a André Wogenscky, 27 de Febrero de 1953, FLC N4.1.38.

${ }^{39}$ Linnanmäki, S., Aalto's Ideas on Air-Conditioning - How Finland Became a "Fanland"?

${ }^{40}$ Dupuy, R., et al. Régulation automatique; Monnier, M., Le Corbusier 1955-2005, Rezé-les-Nantes.
} 
mismo y de su situación en el trayecto diario de los habitantes en el edificio. Como demuestra el proyecto de instalación de la Maison du Brésil, el desfase térmico interior-exterior se amortiguaría creando una transición desde las habitaciones calefactadas a $18{ }^{\circ} \mathrm{C}$, el corredor interior no calefactado, la planta baja calefactada de manera general a $15{ }^{\circ} \mathrm{C}$ y la cámara de transición vidriada con doble puerta de entrada. Por último, la yuxtaposición de múltiples sistemas de calefacción facilitaría una mayor precisión de control térmico, ejemplo que podemos ver en el mismo proyecto: sistema general de calefacción por suelo radiante para garantizar una temperatura de base mínima; radiadores-convectores en zonas más expuestas de planta baja, planta primera y ático; e impulsión de aire acondicionado en el salón de actos.

Al igual que ocurrió con el mur neutralisant respecto a las fachadas en los años 20, el suelo se convirtió durante los años 50 en un elemento de múltiple funcionalidad en la arquitectura de Le Corbusier, sumando a su cometido estructural las dimensiones sensorial térmica, técnica y plástica. El trabajo realizado sobre los solados de edificios como la Maison du Brésil o las Unités d'habitation así lo evidencia. Las plantas residenciales se realizaron en linóleo según la patente Dalami en formato de losetas adheridas de 30x30x1 cm, elegido por su acabado superficial así como por sus propiedades de transmisión térmica y resistencia a dilataciones. Si bien la composición del material con asbestos resulta problemática actualmente por cuestiones sanitarias, en el momento de la construcción fue una propuesta innovadora en la construcción de viviendas. La planta baja, a diferencia, se realizó en solado de pizarra pulida montada según un despiece en bandas paralelas de diferentes dimensiones. La forma de reflejar las luces y de radiar el calor desde la instalación inmediatamente inferior otorgan al suelo de este espacio continuo una presencia térmica y visual que envuelve al usuario. Cabe remarcar, además, que las características formales de tal espacio (continuidad espacial, altura de techo variable y cerramientos curvos) habrían sido difícilmente viables con otro sistema de calefacción. El dispositivo espacial-sensorial descrito no fue una aportación puntual en el trabajo de Le Corbusier, si no que se extendió a otras proyectos de hábitat colectivo, como las Unités d'habitation de Rezé (1949-56), de Briey-en-Forêt (1956-60) y en Firminy (1959-68), o incluso parcialmente en el Convento de la Tourette.

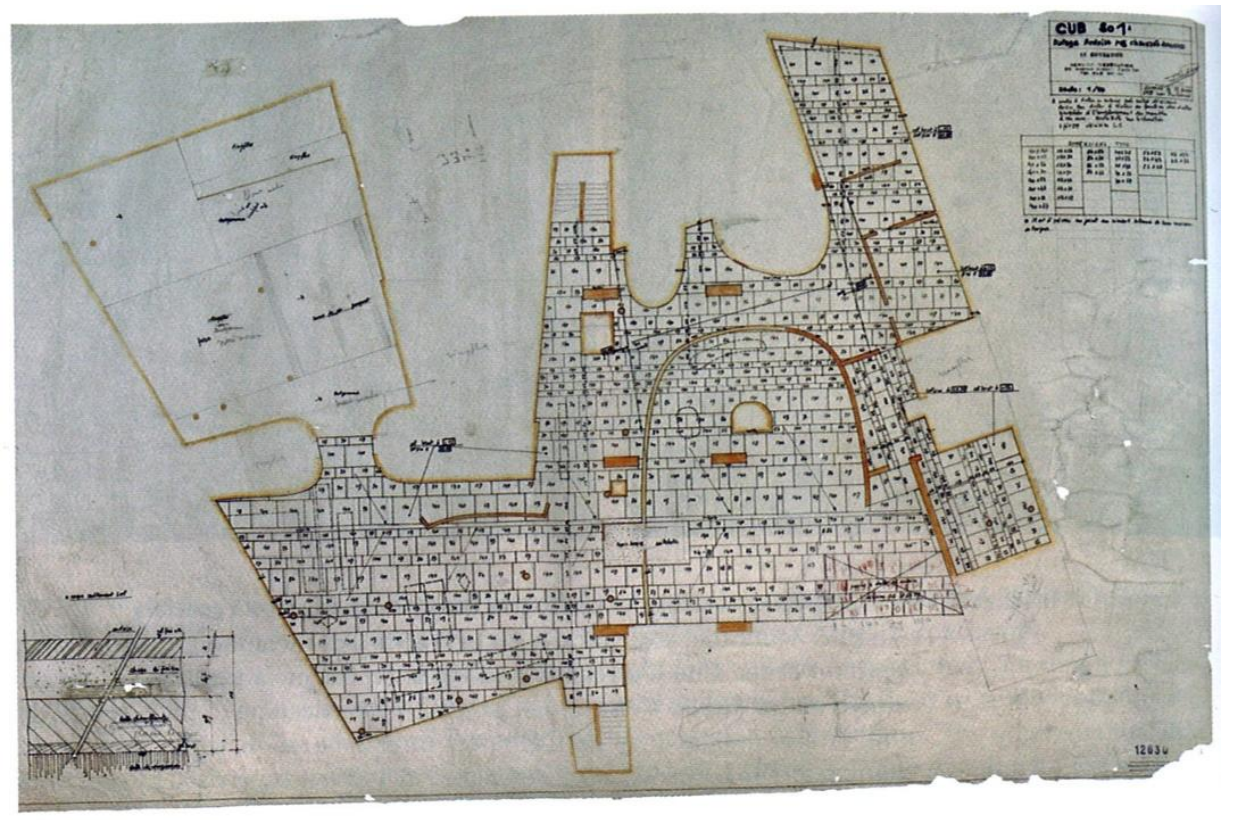

5. Casa de Brasil, planos de despiece de solado de pizarra y trazado de instalación de suelo radiante (FLC 12630). C FLCADAGP. 
La radiación calorífica fue también motivo de experimentación en el proyecto de la Ecole Maternelle de la Unité d'habitation en Firminy. En este caso, la conexión directa del edificio a la red de calefacción urbana fue aprovechada para independizar totalmente el sistema de calefacción en la última planta. La distribución en serie de las aulas sirvió para insertar paneles radiantes integrándolos en la zona inmóvil de las particiones. Los ingenieros se apoyaron en una patente de la Escuela Politécnica de Varsovia con paneles de hormigón conectados directamente a la red. El dispositivo estaba compuesto por un serpentín metálico y una armadura de acero embebidos en el hormigón. Publicado en los Annales del ITBTP en 1957, el citado método aprovechaba la inercia térmica del hormigón al tiempo que permitía explorar el valor plástico del radiador como objeto que formaba parte de las composiciones espaciales de Le Corbusier desde el principio de su practica profesional ${ }^{41}$.

En resumen, la dimensión térmica de las soluciones habitacionales de Le Corbusier en los años 50 revela, de una parte, una interacción más rica y compleja entre clima, arquitectura y cuerpo humano en comparación con sus propuestas de los años 20. Por otra parte, nos indica igualmente una imbricación de las instalaciones de acondicionamiento ambiental en la experiencia espacial y sensorial de la arquitectura de Le Corbusier, evolucionando desde una percepción óculo-céntrica del espacio hacia una percepción más envolvente.

\section{Conclusión: una arquitectura que restablece las condiciones naturales}

Como resultado de un periodo de experimentación conceptual y aplicada a la vez, la comprensión de la arquitectura como una construcción ambiental, especialmente en su dimensión térmica, pasó a formar parte de las propuestas teóricas de Le Corbusier. Desde que en 1945 publicó la primera edición de su teoría de Les Trois Etablissements Humains, la influencia de Winter y Missenard resultaron mas explicitas. En concreto, su interpretación teórica pasó por la afirmación de un medio físico capaz de restituir unas condiciones vitales mas próximas a un ideal natural. Le Corbusier introdujo inicialmente este aspecto bajo el paraguas general de las condiciones de trabajo que, según el, habían desconectado al hombre de su medio primigenio. El clima natural constituía en sus propias palabras un "juego fértil de reacciones favorables al mantenimiento de la máquina humana, muscularmente y nerviosamente (...) La diversidad se imponia, poniendo al hombre en un estado permanente de acomodamiento, de defensa, de mantenimiento, de reforzamiento, de recuperación" ${ }^{\text {"42 }}$. Si Les trois établissements humains pretendía resolver eficazmente los problemas de ocupación del territorio de la civilización maquinista, el restablecimiento de las Conditions de nature en el marco vital del ciudadano permitiría fabricar las condiciones ambientales idóneas para el individuo moderno.

El arquitecto suizo-francés desarrolló este punto de vista en mayor profundidad en el artículo "Rétablir les conditions nature", publicado en 1957 en la revista de paisajismo Espaces verts et jardins. En él, Le Corbusier comenzó explicando cómo la técnica había esclavizado al ser humano determinando un nuevo entorno vital. Una idea anteriormente preconizada por Missenard, quien trató de explicar los peligros del uso descontrolado de las técnicas de acondicionamiento ambiental: "conviene levantarse contra la tendencia actual de realizar climas artificiales de una manera desordenada (...) combatiendo los efectos nefastos de las condiciones artificiales o arbitrarias en las que la civilización material, o la colonización, sitúan al organismo, ${ }^{, 43}$. Le Corbusier, sin embargo, no se apoyó en trabajos ajenos para defender tal conclusión, si no que para él era el resultado de

\footnotetext{
${ }^{41}$ Nam, S., Le radiateur chez Le Corbusier.

${ }^{42}$ Le Corbusier, Les trois établissements humains, p. 63.

${ }^{43}$ Cita original: "il convient de s'élever contre la tendance actuelle à réaliser des climats artificiels d'une façon désordonnée (...) en combattant les effets fâcheux des conditions artificielles ou arbitraires dans lesquelles la civilisation matérielle, ou la colonisation, place l'organisme”. Cf. Missenard, A., L'homme et le climat, p. 253.
} 
"cincuenta años de investigaciones en arquitectura y urbanismo donde se sucedieron y mezclaron el buen 'Hombre', su hogar, su trabajo, su tiempo libre, su ciudad, su cuerpo, su espíritu y su corazón"44. Como resultado, el arquitecto sostuvo el restablecimiento de las condiciones naturales, lo cual aparece en el texto a modo de evolución de cinco postulados ya presentes en la Ville Radieuse: les 24 heures solaires, le sport au pied des maisons, la nature est entrée dans le bail, la ville verte y les techniques modernes s'y prêtent.

El texto considera la necesidad de restablecer las condiciones ambientales próximas a la naturaleza como parte del proyecto urbano global y no exclusivamente de los espacios de trabajo. El hábitat, en consecuencia, se encuentra directamente afectado por la idea de Rétablir les conditions de nature en calidad de elemento creador de ese medio idealizado. De manera diferente a Missenard, para Le Corbusier la naturaleza debía entrar en la vivienda permitiendo crear la impresión de vivir integrado en ella, para lo cual su propuesta pasaba por maximizar el soleamiento directo, vincular la vista al paisaje, favorecer la renovación del aire interior y controlar su temperatura respecto a las variaciones del clima exterior.

En Julio de 1961, Le Corbusier impartió una conferencia en la Facultad de Medicina de París, titulada "Conditions nature, urbanisme efficace et efficient". El arquitecto preparó una conferencia generalista, explicando su contribución personal a la interacción entre la arquitectura y el clima exterior. Véase por ejemplo la presentación del brise-soleil y los problemas derivados al no utilizarlo, ejemplificados en los edificios de la ONU y de la UNESCO, o su propuesta de ventilación natural para el Secretariado de Chandigarh. Posteriormente, el texto de la conferencia ensalza de nuevo el contraste existente entre los ritmos naturales de la vida, variables y adaptativos, respecto al ritmo único y continuo de la máquina. En palabras de Le Corbusier: "la alternancia que es un fenómeno de naturaleza humana. Todo lo que es humano es alternante: el marchar, el parpadeo de los ojos, la palabra, los labios que hablan, todo lo que ustedes quieran, me da igual, es alternante, mientras que la máquina, ella, es continua "45. Afirmación que, una vez más, podemos considerar análoga al texto de Missenard en 1937: "en una época donde podemos considerar realizar, artificialmente, todos los climas, es conveniente llamar la atención sobre el peligro y la responsabilidad que comporta la generalización, sin precauciones, de estas condiciones artificiales ahora, en permanencia, lo que consideramos estar en el confort ideal "46. En consecuencia, el hábitat se convirtió en un método para la creación de climas artificiales, considerados como ambientes heterogéneos capaces de estimular e interactuar con el cuerpo y la percepción de los habitantes.

La publicación póstuma del libro Mise au point por Jean Petit en $1965^{47}$, sirvió para insistir en la importancia del argumento de restablecer las condiciones naturales como eje fundamental de sus propuestas arquitectónicas y urbanas. La arquitectura moderna tenía para Le Corbusier la responsabilidad de luchar contra la progresiva desnaturalización del entorno vital del ser humano debida al mal uso de las técnicas modernas, entre ellos los sistemas de acondicionamiento ambiental. Su colaboración con Winter y Missenard, unida a la creciente necesidad de vivienda tras la Segunda Guerra Mundial, hizo de su obra un campo de experimentación donde el

\footnotetext{
${ }^{44}$ Le Corbusier, Rétablir les conditions de nature.

${ }^{45}$ Cita original: "l'alternance qui est un phénomène de nature humaine. Tout ce qui est humain est alternatif: la marche, le battement des yeux, la parole, les lèvres qui parlent, tout ce que vous voudrez, ça m'est égal, c'est alternatif alors que la machine, elle, est continue”. Cf. Le Corbusier, "Conditions de nature, urbanisme efficace et efficient", Conferencia en la Facultad de Medicina de París, Julio de 1961, FLC C3.10.46.

${ }^{46}$ Cita original: "À une époque où l'on envisage de réaliser, artificiellement, tous les climats, il convient donc d'attirer l'attention sur le danger et la responsabilité que comporte la généralisation, sans précautions, de ces conditions artificielles maintenant, en permanence, ce qu'on estime être le confort idéal'. Cf. Missenard, A., L'homme et le climat.

${ }^{47}$ Le Corbusier, Mise au point.
} 
objetivo era construir un hábitat, en sus dimensiones sensorial, ambiental y física, que transcendiera la función básica de alojamiento y cumpliera un objetivo social más amplio en el conjunto del proyecto de la modernidad.

\section{Agradecimientos}

Los autores desean agradecer a la Fondation Le Corbusier y a la Bibliothèque Nationale de France por el acceso a sus fondos documentales. La presente investigación ha sido financiada con el apoyo de la Fondation Le Corbusier - Bourses pour Jeunes Chercheurs 2013.

\section{Bibliografía/referencias}

Ackermann, Marsha E.: Cool Comfort: America's Romance with Air-Conditioning. Washington: Smithsonian Institution Press, 2002.

Campbell, Margaret: "What Tuberculosis Did for Modernism: The Influence of a Curative Environment on Modernist Design and Architecture". En Medical History. Octubre 2005, n 49. pp. $463-88$.

Cooper, Gail: Air-Conditioning America: Engineers and the Controlled Environment, 1900-1960. Baltimore: The John Hopkins University Press, 1998.

Corbin, Alain: La pluie, le soleil et le vent : une histoire de la sensibilité au temps qu'il fait. Paris: Aubier, 2013.

De la Soudière, Martin: Au bonheur des saisons. Voyage au pays de la météo. Paris: Grasset, 1999.

Dupuy, R.; Broida, V.; Marmillot, A.; Touzard, P.; Raussou, J: Régulation automatique. Annales de l'Institut Technique du Batiment et des Travaux Publiques. N 60. 1952.

Fleming, Rodger; Jankovic, Vladimir: "Revisiting Klima". En Osiris. $\mathrm{N}^{\circ}$ 26. Chicago: The University of Chicago Press, 2011. pp. 1-16. doi:10.1086/661262.

Hobday, Richard: The Light Revolution: Health, Architecture, and the Sun. Forres: Findhorn Press, 2006.

Le Corbusier: "Aux approches d'une synthèse. Aboutissement de vingt années consacrées à la recherche d'une doctrine du domaine bâti". En Boesinger, Willy; Le Corbusier. (Ed.): Le Corbusier. CEuvre Complète 19381946. Zurich: Les éditions d'architecture, 1947. $7^{\mathrm{a}}$ ed. 1977. pp. 66-68.

Le Corbusier: La ville radieuse: eléments d'une doctrine d'urbanisme pour l'équipement de la civilisation machiniste. Boulogne-sur-Seine: L'Architecture d'aujourd'hui, 1935.

Le Corbusier: Les trois établissements humains. Paris: Editions Denoël, 1945.

Le Corbusier: Mise au point. Paris: Forces Vives, 1965.

Le Corbusier: "Rétablir les conditions de nature". En Espaces verts et jardins, $N^{\circ}$ 7. 1957. pp. 33-43.

Le Corbusier: Vers une architecture. París: Crès, 1923.

Linnanmäki, Seija: “Aalto's Ideas on Air-Conditioning - How Finland Became a 'Fanland'?". En Working papers - Alvar Aalto Researchers' Network. Seinäjoki: Alvar Aalto Museum, 2012.

Marino, Giulia: “'Some Like It Hot!' Le confort physiologique et ses dispositifs dans l'architecture du XXè siècle : histoire et devenir d'un enjeu majeur." Director: Franz Graff. École Polytechnique Fédérale de Lausanne, Faculté de l'environnement natural, architectural et construit, 2014.

Missenard, André: Le chauffage et le rafraichissement par rayonnement. París: Eyrolles, 1959.

Missenard, André: À la recherche de l'homme. París: Librairie Istra, 1954.

Missenard, André: À la recherche du temps et du rythme. París: Plon, 1940.

Missenard, André: L'homme et le climat. París: Librairie Plon, 1937. 
Missenard, André: "Le conditionnement des locaux. Conceptions et réalisations modernes". En La technique moderne. $\mathrm{N}^{\circ}$ 7. 1934.

Monnier, Marilyne: Le Corbu 1955-2005 Rezé-Les-Nantes. Rezé: Marilyne Monnier, 2005.

Nam, Sung-Taeg: "Le radiateur chez Le Corbusier". En Massilia 2012. Annuaire d'études corbuséennes. La boite à miracles - Le Corbusier et le théâtre. Marsella: Éditions Imbernon, 2012. pp. 17-42.

Requena-Ruiz, Ignacio; Siret, Daniel: "Experiments on Thermal Comfort and Modern Architecture: The Contributions of André Missenard and Le Corbusier". En Rosso, Michela. (Ed.): Third EAHN International Meeting. Investigating and Writing Architectural History: Subjects, Methodologies and Frontiers. Torino: Politecnico di Torino, 2014. pp. 651-62.

Sadar, John: "The Healthful Ambience of Vitaglass: Light, Glass and the Curative Environment". Architectural Research Quaterly. $\mathrm{N}^{\circ}$ 12. 2008. pp. 269-81. doi:10.1017/S1359135508001206.

Simonnet, Cyrille: Brève histoire de l'air. Versailles: Editions Quæ, 2014.

Siret, Daniel: "Grille climatique, 1951". En Fondation Le Corbusier (Ed.). Le Corbusier Plans, vol. 11. Paris: Echelle-1 \& Fondation Le Corbusier, 2005.

Siret, Daniel: "Les sensations du soleil dans les théories architecturales et urbaines : de l'hygiénisme à la ville durable”. En Krampl, Ulrike; Beck, Robert; Retaillaud-Bajac, Emmanuelle. (Eds.). Les cinq sens de la ville du moyen âge à nos jours. Tours: Presses universitaires François-Rabelais (PUFR), 2013. pp. 105-117.

Sloterdijk, Peter: Esferas III - Espumas, esferología plural. Madrid: Ediciones Siruela, 2006.

Torres Cueco, Jorge: Pensar la arquitectura: Mise au point de Le Corbusier. Madrid: Abada Editores, 2014. 